\title{
Changing process of pharmacy practice: A world-wide perspective
}

\author{
Yara Abu Taleb \\ Royal Medical Services, Amman, Jordan
}

Correspondence author e-mail: y abutaleb@yahoo.com

\begin{abstract}
Objectives: To discuss and critically analyze the general principles of pharmacy practice worldwide and to give the reader sufficient background information on pharmacy profession, and a practical guidance to those concerned in providing pharmaceutical services and their future developments.

Methods: This paper is based on critical analysis of relevant literature on pharmacy practice including chapters in books and articles from international journals. Another major source of information was the writer's personal experience with different patterns of pharmaceutical services through her work as a pharmacist in the Royal Medical Services of the Jordan Armed Forces.

Conclusions: There is a critical need within pharmacy practice to define the essential pharmaceutical care activities that need to be provided to patients in all types of patient-care settings. Pharmacists must continue to provide the pharmaceutical care to patients based on their drug knowledge.
\end{abstract}

Key words: Pharmacy practice, Pharmaceutical care, Quality of care, Patient's counselling, Pharmacist's knowledge and skills.

\section{INTRODUCTION}

The practice of pharmacy is changing; changing from a drug-oriented practice to a patient- oriented one. In the past pharmacists were satisfied when patients left their pharmacy with the right medicine in the right package and with the right instructions. Pharmaceutical care stopped at the front door of the pharmacy.

Today we see a movement where pharmacists want to extend their care. Nowadays they feel a responsibility for the way their patients respond to therapy, they extend their responsibility to the way patients use their medicines. It goes without saying that this responsibility touches the responsibility of the treating doctor and that the patient only benefits if doctor and pharmacist work closely together.

Pharmaceutical Care Concept

Pharmaceutical care is a concept developed in the USA by Hepler and Strand (1990). In their paper, these authors envisaged the future role of pharmacists as a clinical one with pharmacists co-operating with other health care professionals in designing, implementing and monitoring therapeutic plans designed to produce specific therapeutic outcomes for patients.

Hepler and Strand (1990) have developed a model for pharmacy practice whose goal is to make sure pharmacists have a direct responsibility to patients for the outcomes of drug therapy. Pharmaceutical care is the term used to describe this practice, and their definition is as follows:

"Pharmaceutical care is the responsible provision of drug therapy for the purpose of achieving definite outcomes that improve a patient's quality of life".

These outcomes are:

- Cure of disease.

- Elimination or reduction of a patient's symptomatology.

- Arresting or slowing of a disease process; or 
- Preventing a disease or symptomatology.

Strand (1998), believes that pharmacists have to assume responsibility for providing a service of real value and in a way that they could be paid for it. This responsibility is much more than simply providing a product. If there is a difference between pharmaceutical care and clinical pharmacy, it is the pharmaceutical care, which has a management system, and clinical pharmacy is on the periphery of a system that is essentially a dispensary and being managed as such.

In other words pharmaceutical care involves the process, through which a pharmacist co-operates with a patient and other professionals in designing, implementing, and monitoring a therapeutic plan that will produce specific therapeutic outcomes for the patient. This, in turn, involves three major functions (Strand, 1997):

- Identifying potential and actual drug related problems.

- Resolving actual drug-related problems; and

- Preventing potential drug-related problems

Drug-related problems can occur in the following ways (Hepler, 1990):

- Needing pharmacotherapy but not receiving it (prescribing may be inappropriate).

- Taking or receiving the wrong drug.

- Taking or receiving too little of the correct drug

- Taking or receiving too much of the correct drug.

- Experiencing an adverse drug reaction.

- Experiencing a drug-drug, drug-food, or drug-laboratory interaction.

- Not taking or receiving the drug prescribed.
- Taking or receiving a drug for which there is no valid medical indication; or

- Pharmacists or physicians may not monitor patients appropriately.

Recognising that simply compounding and dispending drug products to patients without the appropriate pharmaceutical care processes are not always effective means of reducing drugrelated problems, the profession evolved clinical functions and responsibilities to maximise the benefits while reducing the risks of drug therapy. Thus, in addition to the compounding and dispensing process, the scope of contemporary pharmacist's activities may now also include (Al-Shakhaa and Zairi, 1998):

- Participating in the process of drug therapy decisions.

- Selecting the drug product dosage form.

- Determining the dose and dosage schedule.

- Selecting the drug product source of supply.

- Preparing the drug product for patient use.

- Providing the drug product to the patient.

- Monitoring the patient to maximise complains with therapy instructions.

- Monitoring to detect adverse reactions and drug interactions; and

- Monitoring the patient to ensure that therapy is proceeding in accordance with established therapeutic objectives.

The objective of these services is the achievement of the previous mentioned outcomes that improve a patient's quality of life. The integration of the services described above with the responsibilities for their effect (outcome) in-patients is the philosophy of 
Pharmaceutical Care. In conclusion Pharmaceutical care directs pharmacists' attentions and efforts towards their results in the patients whom pharmacists serve.

\section{Pharmaceutical Care Concept and Quality}

The Institute of Medicine in USA (IOM) has advanced one definition of quality of care as: "The degree to which health care services for individuals and populations increase the likelihood of desired health outcomes and are consistent with current professional knowledge" (Lohr, 1990).

This definition has much in common with Strand and Hepler's definition of pharmaceutical care and both definitions direct attention to the application of health services for purposes of achieving specific health outcomes.

The emphasis on quality in health care focuses increasing attention on the management processes required in providing services to patients. Pharmaceutical care results from the application of the principles of quality management to the use of medications. Quality management can be summarised as requiring (UKCPA, 1996)

- Dialogue with consumers of care.

- Teamwork among providers of care.

- Process definition and assignment responsibility; and

- Measurement and recording of data to prompt continuous care.

The delivery of pharmaceutical care, therefore, requires a quality system to be established for the individual treatment of patients with medication. By its nature, the care of hospitalised patients has always required the contributions of professionals trained in many different fields, and clearly pharmacy has always played a critical role in the patient care.
It is the function of the multidisciplinary team to implement a system for the overall management of the use of medication in patients. It is the function of the pharmacist to take responsibility for that system of medication usage, which will provide the required level of quality assurance. Quality assurance is the traditional strategy that has been used to enhance quality care.

Mount, (1994) sees that the test of quality assurance in drug therapy is the demonstration that the usage of medication accords with patient's needs. The patient's pharmaceutical care needs can be said to have been met when their drug treatment meets identified objectives in terms of clinical benefits, safety, efficiency of medication usage and patient preferences.

\section{Providing Pharmaceutical Care}

Providing pharmaceutical care entails accepting responsibility for patients' pharmaco-therapeutic outcomes. Pharmacists can contribute to positive outcomes by educating and counselling patients to prepare and motivate them to follow their pharmaco-therapeutic regimens and monitoring plans. In working with individual patients, patient groups, families, and caregivers, pharmacists should approach education and counselling as interrelated activities. Good pharmacy practice believes that pharmacists should educate and counsel all patients to the extent possible, going beyond the minimum requirements of laws and regulations; simply offering to counsel is inconsistent with pharmacists' responsibilities. In pharmaceutical care, pharmacists should encourage patients to seek education and counselling and should eliminate barriers to providing it.

Pharmacists should seek opportunities to participate in health-system patient-education programs and to support the educational efforts of other health care 
team members. Pharmacists should collaborate with other health care team members, as appropriate, to determine what specific information and counselling are required in each patient care situation. A coordinated effort among health care team members will enhance patients adherence to pharmaco-therapeutic regimens, monitoring of drug effects, and feedback to the health system (ASHP, 1989).

\section{Pharmacist's Knowledge and Skills}

Although there have been attempts to promote international cooperation and convergence, the content and duration of pharmacy courses vary greatly between countries. In most countries there has been a shift within the curriculum away from chemistry and biology, to a more clinical and social emphasis. The Scandinavian countries, the UK and the Netherlands were the first to incorporate clinical pharmacy into their curricula. There are however, still countries within Europe whose pharmacy education does not encompass clinical pharmacy. Many countries now also teach social pharmacy, including communication skills, as a separate subject area (Taylor and Harding, 2001).

Herrier and Boyce (1995), suggest that in addition to a current knowledge of pharmaco-therapy, pharmacist should know about their patients' cultures, especially health and illness beliefs, attitudes, and practices. They should be aware of patients' feelings toward the health system and views of their own roles and responsibilities for decision-making and for managing their care.

Foster et al., (1995) noted that effective, open-ended questioning and active listening are essential skills for obtaining information from and sharing information with patients and pharmacists have to adapt messages to fit patients' language skills and primary languages, through the use of teaching aids, interpreters, or cultural guides if necessary. Pharmacists also need to observe and interpret the nonverbal messages (e.g., eye contact, facial expressions, body movements, vocal characteristics) patients give during education and counselling sessions.

\section{The Effect of the Environment}

Education and counselling should take place in an environment conducive to patient involvement, learning, and acceptance-one that supports pharmacists' efforts to establish caring relationships with patients. Individual patients, groups, families, or caregivers should perceive the counselling environment as comfortable, confidential, and safe. Education and counselling are most effective when conducted in a room or space that ensures privacy and opportunity to engage in confidential communication. If such an isolated space is not available, a common area can be restructured to maximize visual and auditory privacy from other patients or staff. Patients, including those who are disabled, should have easy access and seating. Space and seating should be adequate for family members or caregivers (ASHP, 1989).

The design and placement of desks and counters should minimize barriers to communication. Distractions and interruptions should be few, so that patients and pharmacists can have each other's undivided attention. The environment should be equipped with appropriate learning aids, (e.g. graphics, anatomical models, medication administration devices, memory aids, written material, and audiovisual resources)

\section{Pharmacists and Patients Roles}

Pharmacists and patients bring to education and counselling sessions their own perceptions of their roles and 
responsibilities. For the experience to be effective, the pharmacist and patient need to come to a common understanding about their respective roles and responsibilities. It may be necessary to clarify for patients that pharmacists have an appropriate and important role in providing education and counselling. Patients should be encouraged to be active participants.

The pharmacists' role is to verify that patients have sufficient understanding, knowledge, and skill to follow their pharmaco-therapeutic regimens and monitoring plans. Pharmacists should also seek ways to motivate patients to learn about their treatment and to be active partners in their care.

Patients' role is to adhere to their pharmaco-therapeutic regimens, monitor for drug effects, and report their experiences to pharmacists or other members of their health care teams, optimally, the patient's role should include seeking information and presenting concerns that may make adherence difficult.

Depending on the health systems' policies and procedures, its use of protocols or clinical care plans, and its credentialing of providers, pharmacists may also have disease management roles and responsibilities for specified categories of patients. This expands pharmacist's relationships with patients and the content of education and counselling session.

\section{The Documentation Process}

Pharmacists should document education and counselling in patients' permanent medical records as consistent with the patients' care plans, the health systems' policies and procedures. When pharmacists do not have access to patients' medical records, education and counselling may be documented in the pharmacy's patient profiles, on the medication order or prescription form, or on a specially designed counselling record.

The pharmacist should record that counselling was offered and was accepted and provided or refused and the pharmacist's perceived level of the patients' understanding. As appropriate, the content should be documented. All documentation should be safeguarded to respect patient confidentiality and privacy and to comply with applicable laws.

\section{CONCLUSION:}

The vision of pharmacy's future is now shifting to a system of drug use in which pharmacists share with other healthcare professionals the responsibility for optimising the outcomes of patients' drug therapy. This role requires pharmacists to apply a higher level of drug knowledge, clinical skill, and independent judgement to their work and to accept a greater amount of responsibility for it.

Pharmaceutical care is an excellent mission and no one seems to disagree with the principles. The challenge is implementation. Any model of pharmacy practice that describes itself as pharmaceutical care must be comprehensive and integrate all functions and responsibilities that are both necessary and sufficient for accomplishing the desired outcomes.

\section{REFERENCES}

Al-Shakhaa, W. and Zairi, M. (1998), Delivering effective health care through teamwork: the pharmaceutical management, Health Manpow Manage. 24(6):212-221.

ASHP, (1989), ASHP statement on the pharmacist's clinical role in organized health- care settings, Am. J. Hosp. Pharm., 46, 805-806.

Foster, SL., Smith, EB., and Seybold MR., (1995), Advanced counselling 
techniques: integrating assessment and intervention. Am Pharm., NS35, 40- 8. quiz 48-50

Hepler, C. (1990), The future of pharmacy: pharmaceutical care, American Pharmacy, NS30, 583-589.

Hepler, C.D. and Strand, L. (1990), Opportunities and responsibilities in pharmaceutical care, American Journal of Hospital Pharmacists, 46, 533-543.

Herrier, RN., and Boyce RW., (1995), Does counselling improve compliance? Am Pharm. NS35, 11- 2, 61

Lohr, K. ((Ed.) 1990), Medicare: A Strategy for Quality Assurance, Vol. 1, National Academy Press, Washington,
Mount, J., (1994), The quality revolution and pharmacy practice, American pharmacy, 6, 52-70.

Strand, L. (1997), Pharmaceutical care: the Minnesota model, Pharmaceutical Journal, 258, 899-904.

Strand, L. (1998), Building a practice in pharmaceutical care, Pharmaceutical Journal, 260, 874-876.

Taylor Kevin and Harding Geoffrey, (2001). The development of pharmacy practice, Pharmacy Practice. Taylor and Francis, London. pp 4-30.

UKCPA, (1996), Statement on Pharmaceutical Care, The Pharmaceutical Journal, 256, 345-346.

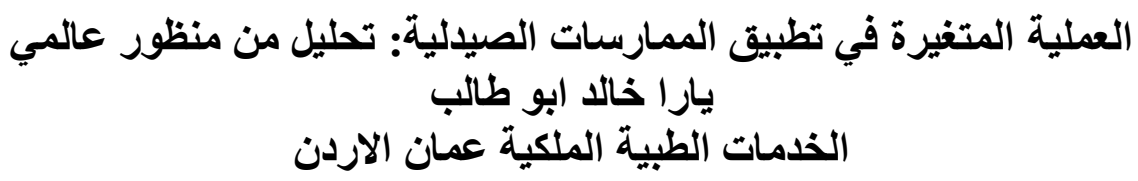

الأهداف :مناقثة وتحليل نقاي للمبادئ العامة لممارسة الصيدلة في جميع أنحاء العالم، وإعطاء القارئ

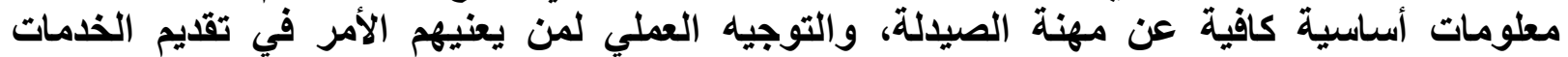

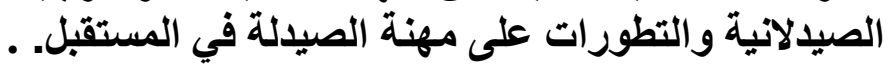

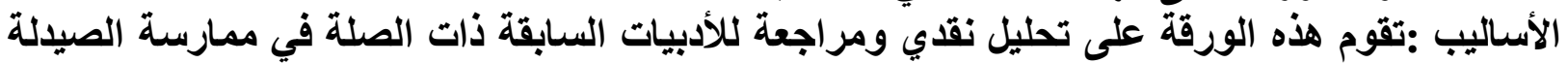

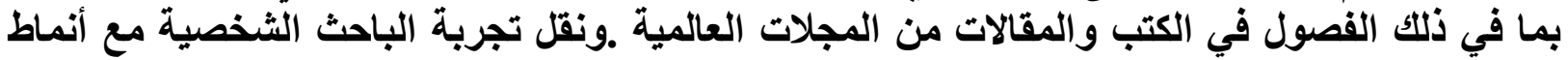
مختلفة من الخدمات الصيدلانية من خلال عملها كصيدلانية في الخدمات الطبية الملكية في القوات التئ المسلحة

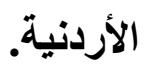
الاستتتاجات :توصل الباحث ان هنالك حاجة ماسة عند ممارسة مهنة الصيدلة لتحديد أنثطة الرعاية

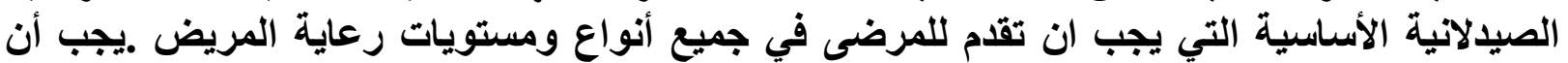

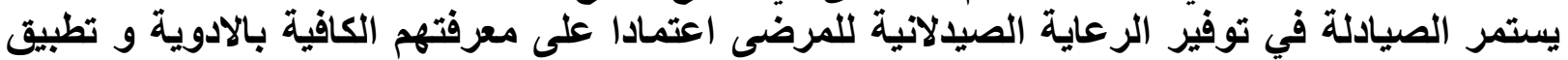

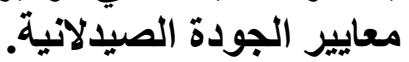

